\title{
Avaliação da atividade antimicrobiana da planta hyptis pectinata: Uma revisão da literatura sistemática
}

\author{
Assessment of the antimicrobial activity of the planta hyptis pectinata: A systematic literature \\ review
}

Evaluación de la actividad antimicrobiana de la planta hyptis pectinata: Revisión de la literatura sistemática

\section{Resumo}

A população apresenta conhecimentos sobre ervas medicinais, devido ao hábito de cultivo das plantas nas áreas residências e a transmissão de conhecimento etnobotânico entre indivíduos da comunidade, com finalidade de tratamento de doenças. A Hyptis Pectinata Possui características aromáticas, e pode ser cultivada no quintal das residências, apesar de seu crescimento acontecer de forma natural em volta as matas. Este estudo refere-se à uma revisão de literatura sistêmica sobre o potencial antimicrobiano a partir dos extratos da planta Sambacaité (Hyptis pectinata), com busca de materiais nas bases de dados SCIELO e PUBMED, utilizando as recomendações do Preferred Reporting Items for Systematic Reviews and Meta-Analyses (PRISMA). Foi realizado a seleção de pesquisas em três etapas (leitura de título e resumo, leitura das publicações por completo e aproveitamento dos artigos selecionados para a formação e compilação dos dados). O resultado obtido neste estudo apresenta resultados eficazes de extrato da planta $\mathrm{H}$. Pectinata para efeitos antimicrobianos antiinflamatórios, antioxidante, antinociceptivos e antiedematogênicos.

Palavras-chave: Antimicrobianos; Hyptis; Lamiaceae.

\begin{abstract}
The population presents knowledge about medicinal herbs, due to the habit of cultivating plants in the residential areas and the transmission of ethnobotanical knowledge among individuals in the community, with the purpose of treating diseases. Hyptis pectinata has aromatic characteristics, and can be cultivated in the backyard, although it grows naturally around forests. This study refers to a systemic literature review about the antimicrobial potential from the extracts of the plant Sambacaite (Hyptis pectinata), with search of materials in the SCIELO and PUBMED databases, using the recommendations of the Preferred Reporting Items for Systematic Reviews and Meta-Analyses (PRISMA). Research selection was carried out in three stages (reading the title and abstract, reading the publications in full, and using the selected articles to form and compile the data). The results obtained in this study, presents effective results of $H$. Pectinata plant extract for anti-inflammatory, antioxidant, antinociceptive and antiedematogenic antimicrobial effects.
\end{abstract}

Keywords: Antimicrobial; Hyptis; Lamiaceae.

\section{Resumen}

La población tiene conocimientos sobre hierbas medicinales, debido a la costumbre de cultivar plantas en las zonas residenciales y a la transmisión de conocimientos etnobotánicos entre los individuos de la comunidad para el tratamiento de enfermedades. La Hyptis Pectinata tiene características aromáticas, y puede cultivarse en el patio de las residencias, aunque su crecimiento se produce de forma natural en torno a los bosques. Este estudio se refiere a 
una revisión bibliográfica sistémica sobre el potencial antimicrobiano de los extractos de la planta Sambacaité (Hyptis pectinata), con búsqueda de materiales en las bases de datos SCIELO y PUBMED, utilizando las recomendaciones del Preferred Reporting Items for Systematic Reviews and Meta-Analyses (PRISMA). La selección de las investigaciones se realizó en tres etapas (lectura del título y el resumen, lectura de las publicaciones en su totalidad y utilización de los artículos seleccionados para la formación y recopilación de datos). Los resultados obtenidos en este estudio, presentan resultados efectivos del extracto de la planta H. Pectinata para efectos antiinflamatorios, antioxidantes, antinociceptivos y antimicrobianos antiedematosos.

Palabras clave: Antimicrobiano; Hyptis; Lamiaceae.

\section{Introdução}

O gênero Hyptis expõe uma grande variedade de estudos com ações antimicrobiana, antifúngica, citotóxica, antiinflamatória, anti-HIV e inseticida, devido ao seu metabolismo extraordinário, possuindo características terapêuticas, principalmente em óleos essenciais. o quais são altamente valorizados em inúmeras comunidades que fazem uso de plantas medicinas. Porém, mesmo possuindo numerosas vantagens, o número de pesquisas voltadas à exploração sustentável das espécies pertinente ao gênero, são baixas (Oliveira et al., 2011).

A Sambacaité, cientificamente chamada de Hyptis pectinata, apresenta origem Americana no Oeste Indiano e Oeste Africano. Esta planta pode ser encontrada facilmente em estradas e matas no estado de Sergipe, devido ao seu crescimento que acontece de forma natural, especialmente em estações chuvosas (Feitosa, 2013). Os vendedores de plantas medicinais (raizeiros), também comercializam a Sambacaité por ser conhecida popularmente através de suas vantagens para uso medicinal, sendo assim, apresentando também importância econômica (Basílio et al., 2006).

O óleo essencial da planta Hyptis pectinata, popularmente conhecida por Sambacaité, apresenta ótimos resultados em sua atividade antimicrobiana contra bactérias Gram-positivas (Santos et al., 2008). Um dos maiores problemas de saúde pública, considera-se a resistência bacteriana, apresentando decorrências associadas à problemas clínicos e econômicos preocupantes, demonstrando também bactérias Gram-positivas mais resistentes aos antibióticos (Loureiro et al., 2016).

Dessa forma, o estudo é de grande relevância para a população e os profissionais de saúde, que buscam novas alternativas medicamentosas e fitoterápicas para o tratamento de diversas enfermidades como principalmente, ações antibacterianas, por exemplo. Sendo ainda considerado um método vantajoso de cuidado, que além de proporcionar benefícios terapêuticos, combatendo as infecções através da inibição do crescimento de micro-organismos, é uma planta de fácil acesso proporcionando também, custo-benefício para o paciente.

\section{Metodologia}

\section{Métodos}

O estudo refere-se a uma revisão de literatura sistêmica e qualitativa, utilizando as recomendações do Preferred Reporting Items for Systematic Reviews and Meta-Analyses (PRISMA), para a busca de artigos para aquisição de dados, e desenvolvimento da pesquisa (Moher et al., 2009).

\section{Critérios de elegibilidade}

Para a leitura e análise foram incluídos neste estudo, artigos, livros e revistas, publicados de forma completa entre os anos de 2000 a 2020, nos idiomas da língua portuguesa e inglesa, o qual manifestaram assuntos compatíveis com esta temática, como artigos que tratam especificamente do gênero Hyptis e espécie Hyptis Pectinata com propriedades medicinais e seu uso popular. Sendo assim, foram descartados os trabalhos que não atenderam a esses requisitos, não apresentaram os descritores selecionados e descrito no tópico estratégia de busca bibliográfica ou ainda materiais científicos duplicados. 


\section{Estratégia de busca bibliográfica}

As referências bibliográficas foram citadas através das publicações selecionadas que atendiam aos critérios de elegibilidade, esses materiais foram obtidos nas bases de dados SCIELO e PUBMED, aplicando como descritores "Lamiaceae" "Antimicrobianos" "Hyptis".

Figura 1 - Busca de artigos para aquisição de dados e desenvolvimento da pesquisa.
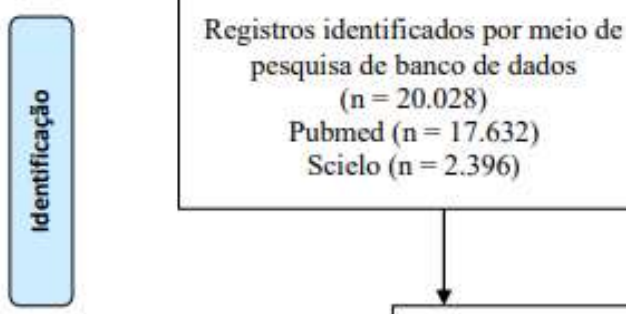
pesquisa de banco de dados $(\mathrm{n}=20.028)$

Pubmed $(\mathrm{n}=17.632)$

Scielo $(\mathrm{n}=2.396)$

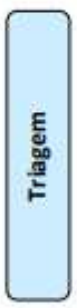

Registros após a remoção de duplicadas $(\mathrm{n}=19.068)$
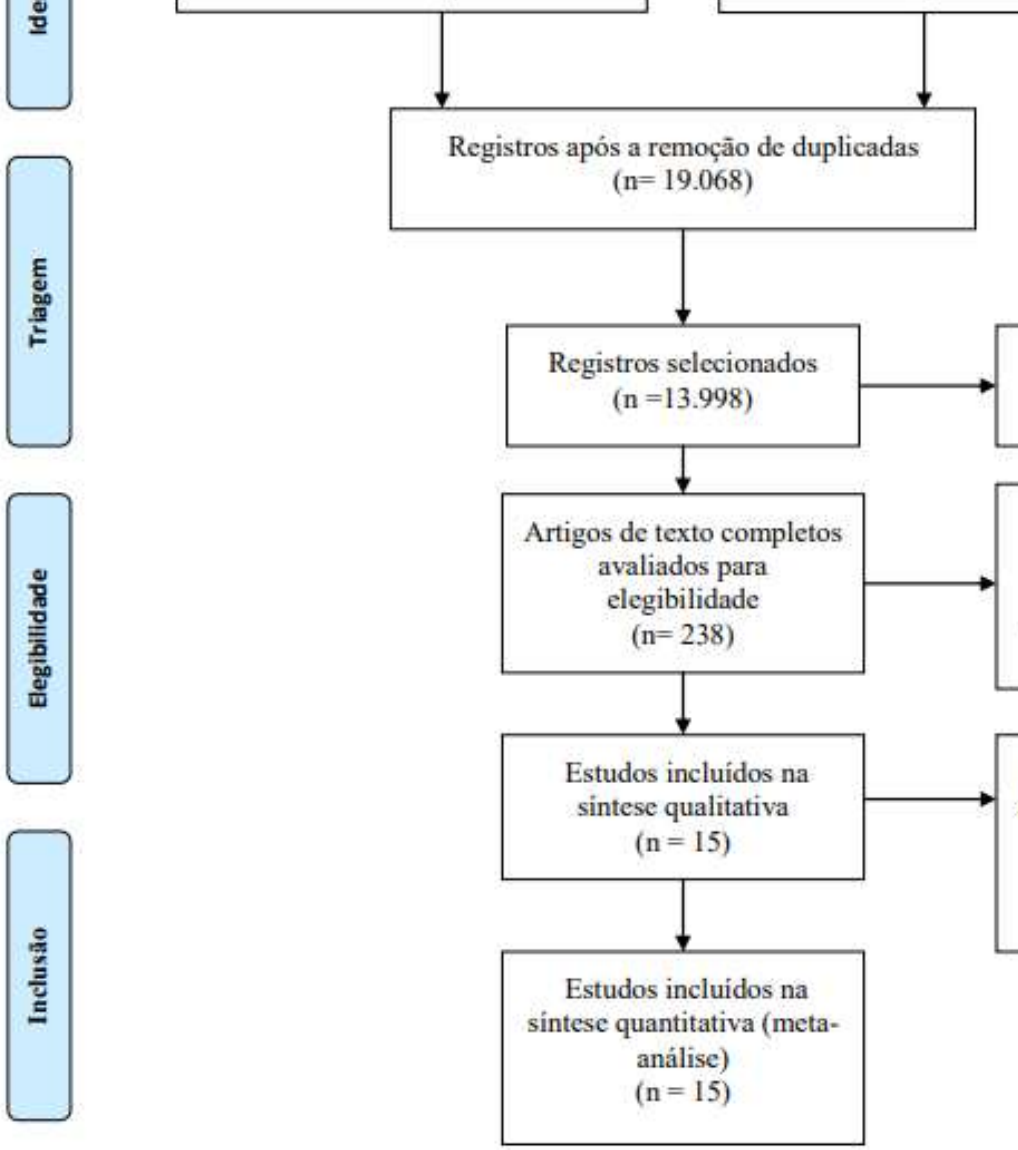

Registros adicionais identificados por outras fontes $(\mathrm{n}=5)$

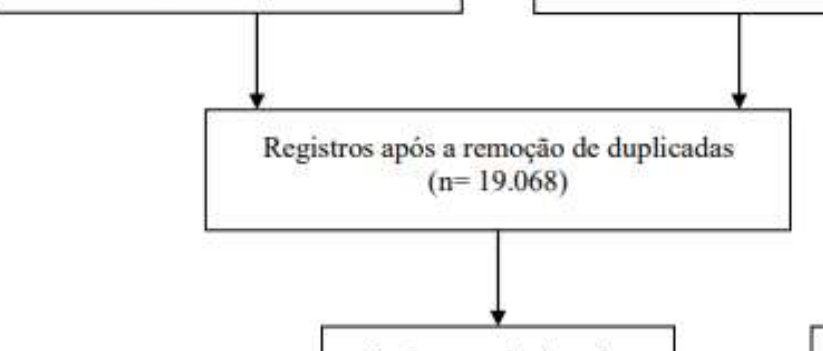

Registros excluidos $(\mathrm{n}=5.070)$

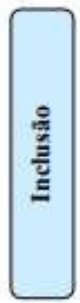
excluidos por não manifestaram assuntos compativeis com a temática. $(\mathrm{n}=13.760)$

Estudos excluidos por não apresentarem especificamente atividades biológicas. $(\mathrm{n}=223)$

Fonte: Moher D, Liberati A, Tetzlaff J, Altman DG, The PRISMA Group (2009). Preferred Reporting Items for Systematic Reviews and MetaAnalyses: The PRISMA Statement. PLoS Med 6(7): e1000097. doi:10.1371/journal.pmed1000097

\section{Seleção dos estudos}

Foi realizado a seleção de pesquisas em três etapas, através da leitura de título e resumos que expressavam ligações ao tema definido descartando os artigos que não atendiam aos critérios de inclusão nas bases de dados. Em seguida, foi realizado a leitura das publicações por completo e a separação de acordo com a melhor correspondência entre os assuntos, com a divisão por posições e análise por etapas, e por último, o aproveitamento dos artigos selecionados para a formação e compilação dos dados. A figura 1, descreve as etapas seguida para a elaboração deste estudo. 


\section{Resultados e Discussão}

Este estudo trata-se de uma revisão sistêmica realizada conforme o modelo Preferred Reporting Items for Systematic Reviews and Meta-Analyses (PRISMA), foram encontrados 20.028 artigos utilizando os descritores: "Lamiaceae" “Antimicrobianos" "Hyptis", e selecionado os estudos que manifestaram melhores relações com o tema. De acordo com os critérios de inclusão e exclusão descritos no estudo, os Quadros 1,2 e 3 representam os resultados obtidos:

Quadro 1 - Resultados de estudos experimentais com Hyptis Pectinata para atividade antimicrobiana, antinociceptiva, aniedematogênica e antioxidante.

\begin{tabular}{|c|c|c|c|c|c|c|}
\hline AUTORES/ANQ & TÍTULOS & \multicolumn{3}{|c|}{ MÉTODOS/MATERIAIS } & \multirow{2}{*}{\multicolumn{2}{|c|}{ RESULTADOS }} \\
\hline \multirow{8}{*}{ Batista, 2015} & \multirow{8}{*}{$\begin{array}{c}\text { Prospecção } \\
\text { Fitoquímica e } \\
\text { Avaliação das } \\
\text { Atividades } \\
\text { Antimicrobiana, } \\
\text { Citotóxica e Anti- } \\
\text { inflamatória de } \\
\text { Hyptis pectinata (L.) } \\
\text { Poit. }\end{array}$} & $\begin{array}{c}\text { AÇÃOO } \\
\text { ANTIMICROBIANA }\end{array}$ & MICRORGANISMOS & ORIGEM & & \\
\hline & & \multirow{7}{*}{$\begin{array}{l}\text { Concentração Mínima } \\
\text { Inibitória (CMI) }\end{array}$} & Staphylococcus aureus AM 103 & ATCC 6538 & & $\mathrm{CMI}=500 \mu \mathrm{g} / \mathrm{mL}$ \\
\hline & & & Staphylococcus aureus AM 106 & ATCC 6538P & & $\mathrm{CMI}=1000 \mu \mathrm{g} / \mathrm{mL}$ \\
\hline & & & $\begin{array}{c}\text { Staphylococcus aureus MSSA } \\
\text { AM } 1235 \\
\end{array}$ & Secreção & & $\mathrm{CMI}=250 \mu \mathrm{g} / \mathrm{mL}$ \\
\hline & & & $\begin{array}{l}\text { Staphylococcus coagulase } \\
\text { negativo AM } 235\end{array}$ & Esperma & & $\mathrm{CMI}=500 \mu \mathrm{g} / \mathrm{mL}$ \\
\hline & & & $\begin{array}{l}\text { Staphylococcus coagulase } \\
\text { negativo AM } 1162\end{array}$ & Fragmento ósseo & & $\mathrm{CMI}=>1000 \mu \mathrm{g} / \mathrm{mL}$ \\
\hline & & & Micrococcus luteus AM 1200 & Líquido peritoneal & & $\mathrm{CMI}=500 \mu \mathrm{g} / \mathrm{mL}$ \\
\hline & & & $\begin{array}{c}\text { Salmonella typhimurium AM } \\
1280\end{array}$ & ATCC 14028 & & $\mathrm{CMI}=125 \mu \mathrm{g} / \mathrm{mL}$ \\
\hline \multirow{13}{*}{$\begin{array}{l}\text { Bispo et al, } \\
\quad 2001\end{array}$} & \multirow{24}{*}{\begin{tabular}{|c|} 
Antinociceptive and \\
antiedematogenic \\
effects of the \\
aqueous extract of \\
Hyptis pectinata \\
leaves in \\
experimental animals
\end{tabular}} & $\begin{array}{c}\text { AÇÃO } \\
\text { ANTINOCICEPTIVA }\end{array}$ & ANIMAIS & TRATAMENTO & $\begin{array}{l}\text { DOSE } \\
\text { (MG / } \\
\text { KG) }\end{array}$ & INIBIÇÃO (\%) \\
\hline & & \multirow{5}{*}{$\begin{array}{l}\text { Teste de contorções } \\
\text { induzidas por ácido } \\
\text { acético e }\end{array}$} & \multirow{16}{*}{ Camundongos Swiss (20-30 g) } & Indometacina & 10 & 47 \\
\hline & & & & Morfina & 2,5 & 66 \\
\hline & & & & \multirow{3}{*}{$\begin{array}{l}\text { Administração oral do extrato } \\
\text { aquosoHG }\end{array}$} & 100 & 43 \\
\hline & & & & & 200 & 51 \\
\hline & & & & & 400 & 54 \\
\hline & & \multirow{7}{*}{ Teste de placa quente } & & TRATAMENTO (MG / KG) & & INIBIÇÃO (\%) \\
\hline & & & & Morfina 10 & & 56,9 \\
\hline & & & & Morfina + naloxona 10 & & 12,28 \\
\hline & & & & EA 100 & & 7,04 \\
\hline & & & & EA 200 & & 47,04 \\
\hline & & & & EA 200 + naloxona & & $-24,82$ \\
\hline & & & & EA 400 & & 37,55 \\
\hline \multirow{11}{*}{$\begin{array}{l}\text { Bispo et al, } \\
\quad 2001\end{array}$} & & \multirow{4}{*}{ Teste de formalina } & & \multicolumn{2}{|l|}{ TRATAMENTO (MG / KG) } & INIBIÇÃO (\%) \\
\hline & & & & Morfina 7,5 & & 100 \\
\hline & & & & Administração oral do extrato aqu & so 200 & 70 \\
\hline & & & & Administração oral do extrato aqu & so 400 & 30,28 \\
\hline & & $\begin{array}{c}\text { AÇÃO } \\
\text { ANIEDEMATOGÊNICA }\end{array}$ & \multirow{7}{*}{ Ratos Wistar (180-240 g) } & \multicolumn{2}{|l|}{ TRATAMENTO (MG / KG) } & INIBIÇÃO (\%) \\
\hline & & \multirow{4}{*}{$\begin{array}{l}\text { Edema induzido por } \\
\text { carragenina }\end{array}$} & & \multicolumn{2}{|l|}{ Indometacina 10} & 36,71 \\
\hline & & & & \multirow{2}{*}{\multicolumn{2}{|c|}{$\begin{array}{l}\text { Administração oral do extrato aquoso } 600 \\
\text { Administracão oral do extrato aquoso } 200\end{array}$}} & 6,38 \\
\hline & & & & & & 5,67 \\
\hline & & & & \multicolumn{2}{|c|}{ Administração oral do extrato aquoso 400} & 34,1 \\
\hline & & \multirow{2}{*}{$\begin{array}{l}\text { Edema induzido por } \\
\text { ácido araquidônico }\end{array}$} & & NDGA $(100 \mathrm{mg} / \mathrm{kg})$ & & 63,42 \\
\hline & & & & \multirow{2}{*}{\multicolumn{2}{|c|}{$\begin{array}{l}\text { CONCENTRAÇÕES FINAIS DE EXTRATO } \\
\text { ALCOOLICO }\end{array}$}} & 32,8 \\
\hline \multirow{5}{*}{ COSTA, 2016} & \multirow{5}{*}{$\begin{array}{l}\text { Triagem fitoquímica e } \\
\text { avaliação da } \\
\text { atividade } \\
\text { antioxidante da } \\
\text { espécie Hyptis } \\
\text { pectinata }\end{array}$} & AÇÃO ANTIOXIDANTE & MATERIAL VEGETAL & & & CONSTANTES CINÉTICAS \\
\hline & & \multirow{4}{*}{$\begin{array}{l}\text { Método de descoloração } \\
\text { do radical 2,2-DIFENIL-1-1- } \\
\text { PICRILHIDRAZIL }\end{array}$} & \multirow{4}{*}{ FOLHAS } & 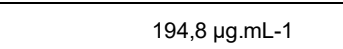 & & $k^{\prime}=2,67 \times 10-4 \mathrm{~min}-1$ \\
\hline & & & & $324,5 \mu \mathrm{g} \cdot \mathrm{mL}-1$ & & $k^{\prime}=2,29 \times 10-4 \mathrm{~min}-1$ \\
\hline & & & & $454,7 \mu \mathrm{g} \cdot \mathrm{mL}-1$ & & $k^{\prime}$ e $\alpha=1,2$ \\
\hline & & & & $584,4 \mu \mathrm{g} \cdot \mathrm{mL}-1$ & & $k^{\prime}$ e $\alpha=1,2$ \\
\hline
\end{tabular}

Fonte: Autoria própria. 
Quadro 2 - Resultados de estudos experimentais com Hyptis Pectinata para atividade antimicrobiana.

\begin{tabular}{|c|c|c|c|c|c|}
\hline AUTORES/ANO & TÍTULOS & \multicolumn{2}{|c|}{ MÉTODOS/MATERIAIS } & \multicolumn{2}{|c|}{ TRATAMENTO } \\
\hline \multirow{14}{*}{ Santos et al, 2008} & \multirow{14}{*}{$\begin{array}{l}\text { Chemical composition and } \\
\text { antimicrobial activity of the } \\
\text { essential oil of Hyptis } \\
\text { pectinata (I.) Poit. }\end{array}$} & $\begin{array}{c}\text { AÇÃO } \\
\text { ANTIMICROBIANA }\end{array}$ & MICROORGANISMOS & $\begin{array}{c}\mathrm{MIC}(\mathrm{mg} \\
\mathrm{mL}-1)\end{array}$ & \begin{tabular}{|c|} 
MFC e \\
MBC \\
$\left(\begin{array}{c}\mathrm{mg} \mathrm{mL} \\
1)\end{array}\right.$ \\
\end{tabular} \\
\hline & & \multirow{2}{*}{$\begin{array}{c}\text { Concentração } \\
\text { inibitória mínima } \\
\text { (miC) e }\end{array}$} & $\begin{array}{l}\text { Salmonella enteritidis } \\
\text { ATCC } 25928\end{array}$ & 300 & 300 \\
\hline & & & $\begin{array}{c}\text { Pseudomonas } \\
\text { aeruginosa ATCC } 1238\end{array}$ & 200 & 200 \\
\hline & & \multirow{11}{*}{$\begin{array}{c}\text { concentração } \\
\text { microbicida mínima } \\
(\mathrm{mmC})\end{array}$} & $\begin{array}{l}\text { Klebsiella pneumoniae } \\
\text { ATCC } 13883\end{array}$ & 200 & 200 \\
\hline & & & $\begin{array}{c}\text { Enterococcus faecalis } \\
\text { ATCC } 29212\end{array}$ & 50 & 100 \\
\hline & & & $\begin{array}{l}\text { Bacillus subtilis ATCC } \\
6633\end{array}$ & 12,5 & 12,5 \\
\hline & & & $\begin{array}{c}\text { Staphylococcus aureus } \\
\text { ATCC } 12600 \\
\end{array}$ & 12,5 & 100 \\
\hline & & & $\begin{array}{c}\text { Staphylococcus } \\
\text { epidermidis ATCC } \\
12228 \\
\end{array}$ & 18,75 & 18,75 \\
\hline & & & $\begin{array}{c}\text { Neisseria gonorrhoeae } \\
\text { ATCC } 1942\end{array}$ & 75 & 75 \\
\hline & & & $\begin{array}{l}\text { Candida albicans } \\
\text { ATCC } 18804\end{array}$ & 0,58 & 0,58 \\
\hline & & & $\begin{array}{c}\text { Candida guilhermondii } \\
\text { ATCC } 6260\end{array}$ & 12,5 & 25 \\
\hline & & & $\begin{array}{c}\text { Candida parapsilosis } \\
\text { ATCC } 22019\end{array}$ & 25 & - \\
\hline & & & $\begin{array}{c}\text { Candida dubliniensis } \\
\text { ATCC MYA-646 }\end{array}$ & 3,12 & 3,12 \\
\hline & & & $\begin{array}{c}\text { Cryptococcus } \\
\text { neoformans IOC } 7523\end{array}$ & 1,17 & 1,17 \\
\hline
\end{tabular}

Fonte: Autoria própria.

Quadro 3 - Resultados de estudos experimentais com Hyptis Pectinata para atividade anti-inflamatória.

\begin{tabular}{|c|c|c|c|c|c|c|c|c|c|}
\hline AUTORES/ANO & TíTULOS & MÉTODOS/ & MATERIAIS & TRATAME & NTO (MG / KG) & & INIBICC & ÃO (\%) & \\
\hline \multirow{5}{*}{$\begin{array}{l}\text { Raymundo et al, } \\
2011\end{array}$} & \multirow{5}{*}{$\begin{array}{l}\text { Caracterização das } \\
\text { atividades } \\
\text { antiinflamatórias e } \\
\text { antinociceptivas do } \\
\text { Hyptis pectinata ( L.) } \\
\text { óleo essencial Poit }\end{array}$} & $\begin{array}{c}\text { AÇÃO ANTI- } \\
\text { INFLAMATÓRIA }\end{array}$ & ANIMAIS & $\begin{array}{c}\text { DOSE } \\
\text { (MG / KG) }\end{array}$ & $\begin{array}{l}\text { Proteína total } \\
(\mathrm{mg} / \mathrm{ml})\end{array}$ & NO (M) & $\begin{array}{l}\text { PGE } 2 \text { ( pg } \\
/ \mathrm{ml})\end{array}$ & $\begin{array}{c}\text { IL-6 (pg / } \\
\text { ml) }\end{array}$ & TNF- (U / ml) \\
\hline & & \multirow{4}{*}{$\begin{array}{c}\text { Modelo de bolsa } \\
\text { de ar subcutâneo } \\
\text { (SAP) com } \\
\text { injeção de } \\
\text { Carragenina. }\end{array}$} & \multirow{4}{*}{$\begin{array}{c}\text { CAMUNDONGO } \\
\mathrm{S}\end{array}$} & 200 & $31,8 \pm 13,3$ & $71,5 \pm 9,1$ & $15,3 \pm 7,1$ & $\begin{array}{c}288,9 \pm \\
16,3 \\
\end{array}$ & $498,7 \pm 66,9$ \\
\hline & & & & 10 & $203,9 \pm 31,5$ & $91 \pm 10,8$ & $79,4 \pm 9,9$ & $\begin{array}{c}301,4 \pm \\
44,3\end{array}$ & $450,8 \pm 69,1$ \\
\hline & & & & 30 & $156,3 \pm 24,6$ & $85,1 \pm 11,3$ & $74,1 \pm 11,7$ & $\begin{array}{c}275,8 \pm \\
33,6 \\
\end{array}$ & $441,8 \pm 44,6$ \\
\hline & & & & 100 & $121,6 \pm 19,7$ & $74,7 \pm 9,1$ & $65,8 \pm 12,1$ & $\begin{array}{c}255,1 \pm \\
37,4 \\
\end{array}$ & $399,2 \pm 21,7$ \\
\hline
\end{tabular}


Viera \& leite (2018) enfatizam que a população apresenta conhecimentos sobre as ervas para uso terapêuticos, pois, é um hábito o cultivo das plantas nas áreas de suas residências e a transmissão de conhecimento etnobotânico entre indivíduos da comunidade, com finalidade de tratamento de doenças. além disso, também foi constatado que a comunidade necessita de fontes científicas sobre o uso das ervas para garantir de forma segura a utilização dessas plantas.

A planta Hyptis pectinata é uma erva bastante cultivada no Brasil para o tratamento de inflamações, dores, infecções bacterianas e câncer (Santana et al., 2019). Possui características aromáticas, e pode ser cultivada em seu próprio quintal de casa, mas geralmente seu crescimento acontece de forma natural em volta as matas, diversas pesquisas comprovam que o extrato aquoso de suas folhas possui ricas composições para o uso medicinal (Mann et al., 2002). Na medicina popular, utilizase os óleos essenciais e extratos de ervas para diversas aplicações, dentre as grandes variedades, está incluso o uso antimicrobiano e antissépticos tópicos, há uma admirável quantidade de métodos narrados na literatura para mensurar a atividade antimicrobiana de óleos essenciais (Nascimento, 2008).

O extrato da planta é rico em metabólicos secundários, sendo identificados os respectivos compostos: Taninos, Flavonas, Flavonóis, Xantonas, Catequinas. Os taninos apresentaram um alto teor relacionados a proteína (10,94\%), a fibra (3,22\%), comparando-as com demais espécies, e o total $(1,04 \%)$ quando comparado a outros modelos taniniferos (Costa, 2016). A presença desses compostos, justifica as suas ações biológicas bem divulgadas na literatura e ações farmacológicas atribuídas a esta espécie.

Raymundo \& colaboradores (2011), utilizaram o modelo de CAP (contorções induzidas por ácido acético) em seus experimentos, o qual apresentou resultados satisfatórios do óleo essencial da Hyptis Pectinata para efeitos anti-inflamatórios. Nesse modelo, a carragenina foi aplicada em diferentes concentrações (200mg, 100mg, 30mg, 10mg) como um modelo inflamatório, por injeção subcutânea em camundongos, possibilitando examinar a migração celular e os mediadores inflamatórios obtidos através da administração do óleo por via oral, com resultados descritos no quadro 3.

Em relação à atividade antioxidante, os estudos publicados por Costa (2016), apresentaram resultados positivos. Foram testados para ação antioxidante, os respectivos materiais biológicos: raiz, folhas e caule da espécie Hyptis pectinata, de forma separadamente, por meio do método DPPH (2,2-difenil-1-picril-hidrazil). Menores concentrações (folhas) apontaram atividade antioxidante, menor que $10 \mathrm{mg} / \mathrm{mL}$, sendo mais favoráveis que as concentrações maiores do caule e da raiz, que mesmo manifestando bons resultados, sofreram intervenções da concentração de clorofila presente no extrato, dessa forma, justifica-se também a razão pela qual as folhas da erva serem mais utilizadas nos tratamentos (Costa, 2016).

Nos estudos de Batista (2015), os Microrganismos empregados para determinação da Concentração Mínima Inibitória (CMI) foram cepas bacterianas Gram-positivas (Staphylococcus aureus, Staphylococcus coagulase negativos, Micrococcus luteus), e cepas Gram-negativas (Salmonella sp., Klebsiella pneumoniae, Pseudomonas aeruginosa, Proteus mirabilis, Escherichia coli e Salmonella typhimurium). A explicação desta ação antimicrobiana foi determinada através de três extratos da planta (extrato aquoso, extrato acetato de etila e extrato hexânico), extrato aquoso e acetato de etila exibiram melhores resultados, podendo está relacionado aos compostos fenólicos que os dois possuem.

O extrato aquoso da erva apresentou inibição diante todas as cepas Gram-positivas, sendo Staphylococcus aureus e Staphylococcus coagulase-negativa com efeitos mais expressivos, apresentando CMI entre 250 e $500 \mu \mathrm{g} / \mathrm{mL}$. Ao contrário das cepas Gram-negativas que demonstrou pouca atividade, Klebsiella pneumoniae, Salmonella enterica e Proteus mirabilis expressaram halos de inibição com concentrações de no Max. $10 \mathrm{mg} /$ poço. Com respeito ao acetato de etila, o extrato acetato expressou maiores atividades de CMI frente à Staphylococcus aureus, Staphylococcus coagulase-negativa e Micrococcus luteus com resultados entre 250 e $500 \mu \mathrm{g} / \mathrm{mL}$, em cepas Gram-negativas melhores resultados foram em linhagens de Salmonella sp, com inibição com 125-250 $\mu \mathrm{g} / \mathrm{mL}$. O extrato etanólico de Hyptis pectinata exibiu baixa concentração para efeitos inibitórios (Batista, 2015). 
Santos et al., 2008 afirmam que a planta Hyptis pectinata possui óleo essencial com efeitos satisfatórios contra leveduras e bactérias Gram-positivas (Bacillus subtilis, Enterococcus faecalis, Staphylococcus epidermidis e Staphylococcus aureus), os ensaios apresentaram valores de Concentração Mínima Inibitória (CMI) entre 12,5 e 50 mg mL- 1 para os microorganismos Gram (+), sendo, B. subtilis e $S$. aureus com valores menores (12,5 mg mL-1), e E. faecalis com valor maior (50 mg mL-1). Os resultados para bactérias Gram negativas foram de, $200 \mathrm{mg} \mathrm{mL}-1$ para K. pneumoniae e P. aeruginosa e 300 mg mL- 1 para S. Enteritidis (concentrações são consideradas altas para inibição).

No estado de Sergipe, foram feitos estudos por Bispo et al., 2001 em dois modelos de animais, para determinação de efeitos antinociceptivos e antiedematogênicos do extrato aquoso da $H$. Pectinata. Para a pesquisa antiedematogênicos foram utilizados ratos Wistar de ambos os sexos, pesando de 180 a $240 \mathrm{~g}$, e camundongos Swiss de 20 a $30 \mathrm{~g}$, para as analises antinociceptivos, os animais receberam concetração do extrato entre $400 \mathrm{mg}$ e $2,5 \mathrm{mg}$, respectivamente (quadro 1). Os resultados se deram, através de vários modelos de testes, apresentando os respectivos resultados: testes de contorções abdominais induzidas por ácido acético (entre 43 e 66\%), teste de placa quente (entre 7,04 e 56,9\%), teste de formalina (entre 30,28 e 100\%), edema induzido por carragenina (entre 5,67 e 36,17\%) e edema induzido por ácido araquidônico (entre 32,80 e 63,42).

\section{Considerações Finais}

Tendo em consideração os resultados obtidos neste estudo, pode-se concluir que a planta Hyptis pectinata é eficaz para efeitos antimicrobianos antiinflamatórios, antioxidante, antinociceptivos e antiedematogênicos.

As pesquisas para antimicrobianos apresentaram melhores resultados nos modelos apresentados para óleo essencial, extrato aquoso e acetato de etila, entretanto, os micro-organismos com bactérias gram-negativas tiveram concentrações baixas para inibição, já bactérias Gram-positivas expressou resultados mais satisfatório, com ótimo poder para inibição.

Apesar de a atividade antitumoral ser descrita em alguns estudos etnobotânicos, não foi encontrado nenhum artigo que descrevesse esse tipo de atividade em modelos animais, ou de forma in vitro.

Portanto, independentemente da quantidade de estudos encontrados, ainda se faz necessário o estudo de novas pesquisas, concebendo a população e profissionais de saúde métodos de prevenções e tratamentos seguros e eficazes com $H$. Pectinata.

\section{Referências}

Basílio, I. J. L. D., Agra, M. D., Rocha, E. A., Leal, C. K. A., \& Abrantes, H. F. L. (2006). Estudo farmacobotânico comparativo das folhas de Hyptis pectinata (L.) Poit. E Hyptis suaveolens (L.) Poit. (Lamiaceae). Acta Farm. Bonaerense, 518-525.

Batista, D. C. D. A. (2015). Prospecção Fitoquímica e Avaliação das Atividades Antimicrobiana, Citotóxica e Antiinflamatória de Hyptis pectinata (L.) Poit. Universidade Federal de Pernambuco.

Bispo, M. D., Mourão, R. H. V., Franzotti, E. M., Bomfim, K. B. R., Arrigoni-Blank, M. de F., Moreno, M. P. N., Marchioro, M., \& Antoniolli, A. R. (2001). Antinociceptive and antiedematogenic effects of the aqueous extract of Hyptis pectinata leaves in experimental animals. Journal of Ethnopharmacology, 76(1), 81-86. https://doi.org/10.1016/S0378-8741(01)00172-6

Costa, M. (2016). Triagem fitoquímica e avaliação da atividade antioxidante da espécie Hyptis pectinata. Universidade Federal de Pernambuco.

Falcão, R. E. A. (2013). Avaliação do potencial antioxidante, leishmanicida e antinociceptivodas folhas de Hyptis pectinata(L.)Poit. Universidade Federal Rural de Pernambuco.

Feitosa, R. B. Fenologia, adubação orgânica e micropropagação de acessos de Sambacaitá (Hyptis pectinata L. Poit). [s.1.] Universidade Federal de Sergipe, 2013 .

Loureiro, R. J., Roque, F., Rodrigues, A. T., Herdeiro, M. T., \& Ramalheira, E. (2016). O uso de antibióticos e as resistências bacterianas: Breves notas sobre a sua evolução. Revista Portuguesa de Saúde Pública, 34(1), 77-84. https://doi.org/10.1016/j.rpsp.2015.11.003

Moher D, Liberati A, Tetzlaff J, Altman DG, The PRISMA Group (2009). Preferred Reporting Items for Systematic Reviews and MetaAnalyses: The PRISMA Statement. PLoS Med 6(7): e1000097. doi:10.1371/journal.pmed1000097 
Research, Society and Development, v. 10, n. 7, e42310717066, 2021

(CC BY 4.0) | ISSN 2525-3409 | DOI: http://dx.doi.org/10.33448/rsd-v10i7.17066

Nascimento, P. F.C., Alviano, W. S., Nascimento, A. L.C., Santos, P. O., Arrigoni-Blank, M. F., De Jesus, R. A., Azevedo, V. G., Alviano, D. S., Bolognese, A. M., Trindade, R. C., \& Alviano, W. S. (2008). Hyptis pectinata essential oil: Chemical composition and anti-Streptococcus mutans activity. Oral Diseases, $485-489$.

Oliveira, Nepomuceno, Freitas, Pereira, Silva, \& Lucchese. (2011). Revista Brasileira de Plantas Medicina. Propagação vegetativa de Hyptis leucocephala Mart. ex Benth. e Hyptis platanifolia Mart. ex Benth. (Lamiaceae)is, 13, 73-78.

Raymundo, Larissa J.R.P. Guilhon, Carolina C. Alviano, Daniela S. Matheus, Maria Eline R., Angelo Cavalcanti Alves, Sócrates C.H. Alviano, Péricles B. Fernandes, Celuta S. D., \& Patrícia. (2011). Characterisation of the anti-inflammatory and antinociceptive activities of the Hyptis pectinata (L.) Poit essential oil. Journal of Ethnopharmacology, 725-732.

Santana, F. R., Luna-Dulcey, L., Antunes, V. U., Tormena, C. F., Cominetti, M. R., Duarte, M. C., \& Silva, J. A. da. (2019). Evaluation of the cytotoxicity on breast cancer cell of extracts and compounds isolated from Hyptis pectinata (L.) poit. Natural Product Research, 34.

Santos, Patrícia O., Costa, Marcilene De J.C., Alves, José A.B., Nascimento, Paula F.C., De Melo, Dângelly L.M., Barbosa, Antônio M., Trindade, Rita De C., Blank, Arie F., Arrigoni-Blank, Maria F., Alves, Péricles B., \& Do Nascimento, Maria Da Paz F. (2008). Chemical composition and antimicrobial activity of the essential oil of Hyptis pectinata (1.) Poit. Quimica Nova, 31, 1648-1652.

Silva-Mann, R., Costa, A. G., Silva, P. de A., Mendonça, M. da C., Filho, J. L. S. de C., Dantas, Í. B., Azevedo, V. G., F., M. de, Arrigoni-Blank, \& Blank, A. F. (2003). Avaliação de componentes de produção de sambacaitá no primeiro corte. Horticultura brasileira, 21, 1-4.

Vieira, Vanessa Diniz, Marconi, Lucas, \& Leite, Santos. (2018). O uso do conhecimento popular das plantas medicinais utilizadas pela comunidade no nordeste. Temas em Saúde, 876-890. 